\title{
Actividades lúdicas en las clases de español como lengua extranjera
}

\section{Playful activities in spanish as a foreign language classes}

DOI: http://dx.doi.org/10.17981/cultedusoc.11.2.2020.13

Recibido: 27 de febrero de 2020 Aceptado: 28 de mayo de 2020 Publicado: 09 de julio de 2020

\author{
Ramón Antonio Hernández Chirinos de Jesus \\ Universidad Estadual do Piauí. Porto, Piauí (Brasil) \\ ramon_hernandez2012@hotmail.com \\ Francisco das C Silva de Jesus Hernández \\ Secretaria Municipal de Asistencia Social Porto Piauí. Porto, Piauí (Brasil) \\ professordjesus.2013@yahoo.com.br
}

Para citar este artículo:

Hernández, R. y Silva, F. (2020). Actividades lúdicas en las clases de español como lengua extranjera. Cultura, Educación y Sociedad, 11(2). 207-220. DOI: http://dx.doi.org/10.17981/cultedusoc.11.2.2020.13

\section{Resumen}

El presente trabajo, tiene como objetivo valorar las actividades lúdicas socializadas en las clases de español como lengua extranjera. La investigación se orientó desde una metodología cualitativa que busca generar procesos de comprensión de la realidad, en las que está inmersa la población; para ello, se conformó un grupo con estudiantes del primer año de educación media en una escuela pública de Brasil, cuyas edades oscilaban entre 14 y 20 años. En el estudio se aplicaron como instrumentos, el guión de entrevista y la observación no participante. Los resultados evidencian el papel que cumplen las actividades lúdicas como estrategias pedagógicas para fortalecen las clases de español como lengua extranjera. Se concluye que dichas actividades permiten a los estudiantes un disfrute de la asignatura, consolidándose la adquisición de la lengua con el uso de vocabulario y su puesta en práctica en un contexto real.

Palabras clave: Actividades lúdicas; español lengua extranjera; estrategias pedagógicas: enseñanza y aprendizaje de español

\begin{abstract}
The present work has the objective of evaluating the recreational activities socialized in the classes of Spanish as a foreign language. The research was oriented from a qualitative methodology that seeks to generate processes of understanding of reality, in which the population is immersed;To do this, a group was formed with students from the first year of secondary education in a public school in Brazil, whose ages ranged from 14 to 20 years. In the study, the interview script and nonparticipant observation were applied as instruments. The results show the role of play activities as pedagogical strategies to strengthen Spanish as a foreign language classes. It is concluded that these activities allow students to enjoy the subject, consolidating the acquisition of the language with the use of vocabulary and its implementation in a real context.

Keywords: Leisure activities; Spanish as a foreign language; pedagogical strategies; teaching and learning Spanish
\end{abstract}




\section{INTRODUCCIÓN}

Actualmente existe una notable preocupación por los docentes que imparten la asignatura de español como lengua extranjera, pues en la mayoría de los casos los estudiantes no se sienten motivados hacia la misma. Esta situación ha generado inquietudes en los docentes, ya que, han tenido que repensar como mejorar su práctica docente.

Esta problemática es muy frecuente en las instituciones públicas dependientes del Estado y Municipios, ya que, en las prácticas pedagógicas de los docentes se hace evidente el poco uso de estrategias que llaman la atención del educando, pues gran parte de los mediadores de aprendizaje se limitan a tener como recursos didácticos, el libro del estudiante y el pizarrón. Lo expuesto precisa la necesidad de generar consciencia en los profesionales formados en español, puesto que los mismos deben empezar a desarrollar estrategias o actividades que no solo llamen la atención del estudiante, sino que al mismo tiempo les permita la adquisición de nuevos conocimientos.

Algunos estudios, desde la experiencia de formación e indagación reflexiva, destacan el papel fundamental que juega el docente en la sociedad. Por lo que, las actividades lúdicas se convierten hoy día en excelentes alternativas que inducen a los mediadores de aprendizaje a dejar de utilizar los métodos tradicionales en su entorno educativo, permitiéndoles explorar, trabajar y desarrollar diferentes habilidades con los estudiantes.

Lo antes expuesto, coloca a los docentes en una situación donde debe superar sus propios desafíos asociados a la nueva era, dándose la posibilidad de repensar en sus prácticas pedagógicas, desarrollando así su creatividad con la implementación de actividades lúdicas que pueden beneficiar a los estudiantes en las clases de diferentes disciplinas, incluidas el español

Las actividades lúdicas favorecen el desarrollo de las aptitudes, las relaciones y el sentido del humor en los estudiantes, llamando la atención en ellos para lograr una motivación en su aprendizaje, convirtiéndose estas, en herramientas estratégicas para el proceso de enseñanza y aprendizaje (Calderón, Marín y Vargas 2015). La aplicación de actividades lúdicas permite que los estudiantes alcancen el aprendizaje esperado de manera atractiva y natural desarrollando sus aptitudes, actitudes, adquiriendo conocimientos significativos, mejorando el autoestima y desarrollo del lenguaje en la lengua extranjera (español).

Al respecto, Silveira \& Barone (1998) afirman que:

Los juegos se pueden usar para una variedad de propósitos dentro del contexto de aprendizaje. Uno de los usos básicos y muy importantes es la posibilidad de desarrollar la autoconfianza. Otro es el aumento de la motivación. Un método efectivo que permite una práctica significativa de lo que se está aprendiendo. Incluso el más simple de los juegos puede utilizarse para proporcionar información objetiva y practicar habilidades, confiriendo destreza y competencia (p.02).

Aprender una lengua extranjera le exige al estudiante varios esfuerzos, así como dedicarse a la adquisición de los aspectos lingüísticos, morfológicos y sintácticos del idioma. Para que eso ocurra de manera placentera, es necesario estudiar con ahínco, 
además de la participación espontánea en las clases en que el mediador de aprendizaje adopte estrategias lúdicas en su proceso de socialización de conocimientos. No solo por parte del docente, sino que el socio de aprendizaje debe cambiar su actitud con relación al mismo, lo que hace necesario, actuar de forma que las actividades posibiliten el despertar del educando por el placer de aprender la lengua extranjera. Desde este referente el presente artículo profundiza en la valoración de las actividades lúdicas que son socializadas en la clase de español como lengua extranjera en la Escuela Municipal Popular Remédiense ubicada en el Municipio Nuestra Señora de los Remedios, Estado de Piauí, Brasil.

\section{REFERENTES TEÓRICOS}

\section{La lúdica en la actividad didáctica}

La lúdica es toda actividad de entretenimiento, que da placer; el acto de jugar, las prácticas con fines recreativos, es la libertad de expresión física y emocional es apertura para nuevos conocimientos a las personas involucradas en ese proceso. Originalmente "lúdico" deriva de la palabra latina "ludus" y significa "jugar, juego", sin embargo, la palabra no se limita a este término y pedagógicamente es mucho más amplia. Es una forma de descubrir el mundo y en algunos casos sirve como instrumentos educativos, que está generalmente asociada a la expresión de ideas y emociones que desarrollan habilidades prácticas, y la estimulación mental y física que a menudo sirven como una forma de ejercicio o como un papel educativo, acompañado de un sentimiento de alegría y conocimiento de que se hace diferente a la vida cotidiana.

Por lo que Santos (2006) expresa:

La palabra lúdica proviene del latín ludus y significa jugar. Esta obra incluye juegos, juguetes y diversiones y también está relacionada con la conducta de quienes juegan y juegan. A su vez, la función educativa del juego brinda una oportunidad para el aprendizaje individual, el conocimiento, el conocimiento y la comprensión del mundo (p. 9).

Según, Texeira (1995) "las actividades lúdicas tienen su importancia en el desarrollo de las competencias humanas de los estudiantes, posibilitando medios adecuados para su desarrollo, físico, motor, emotivo, intelectual y formal" (p. 35).

La lúdica es un proceso de extrema importancia en la educación desde la infancia hasta la fase adulta, ya que, la utilización de la ludicidad permite que el estudiante aprenda de forma práctica, resaltando que el universo lúdico proporciona al ser humano experimentar el hecho educativo de manera atractiva en ese proceso de aprendizaje. La lúdica podría considerarse como un insumo fundamental en el desarrollo de las actividades académicas, permitiendo la oportunidad de apartarse de algún modo de los esquemas tradicionales de enseñanza e integrar nuevos elementos al proceso de aprendizaje.

En este sentido Zúñiga (1998) plantea: 
Será repensar lo que hoy se hace en la pedagogía para descubrir los aportes con que la lúdica puede contribuir para conseguir la aplicación de unos criterios más acordes con los tiempos actuales en que la velocidad de los acontecimientos y las transformaciones exige unos niveles de respuesta casi que inmediatos para estar al ritmo actual del mundo moderno, con una rapidez no imaginada desde la óptica del contexto tradicional con que todavía analizamos (p. 1).

Al respecto, Teixeira (1995) afirma:

Las actividades lúdicas integran las diversas dimensiones de la personalidad: afectiva, motora y cognitiva. Como actividades físicas y mentales que movilizan funciones y operaciones, el juego activa las esferas motrices y cognitivas, y al generar participación emocional, atrae a la esfera afectiva. Por lo tanto, se ve que la actividad lúdica es similar a la acción artística, como un elemento integrador de los diversos aspectos de la personalidad. El ser que juega y juega es también el ser que actúa, siente, piensa, aprende y se desarrolla (p. 23).

El uso de la lúdica sugiere alternativas que proporciona la utilización de recursos placenteros como incentivos al proceso de aprendizaje, posibilitando al socio de aprendizaje un despertar por ese aprendizaje motivador, por su parte, Santos (2006) se refiere al desarrollo del aspecto lúdico que facilita el aprendizaje, el desarrollo intrapersonal e interpersonal, colabora con una buena salud mental, se prepara para un estado interno fértil, facilita el proceso de socialización, comunicación, expresión y construcción del conocimiento.

Por otro lado, los juegos lúdicos motivan al estudiante en su aprendizaje, permitiendo al educador verificar el éxito en el proceso del mismo. Es importante que el educando, sepa cómo ponerlas en práctica en sus clases. Para ello, el profesor debe conocer la realidad del estudiante, cuáles son sus preferencias en la ludicidad para que haya una comprensión mejor de contenidos, con acciones que al estudiante le guste. Las actividades lúdicas son como piezas fundamentales para el desarrollo de las personas y de los grupos sociales, en diferentes espacios, lo cual desencadena una mayor concentración.

En este sentido, Teixeira (1995) refiere que:

El juego presenta dos elementos que lo caracterizan: placer y esfuerzo espontáneo. Se considera placentero, debido a su capacidad de absorber al individuo de una manera intensa y total, creando un clima de entusiasmo. Es este aspecto de la participación emocional lo que lo convierte en una actividad con un fuerte contenido motivacional, capaz de generar un estado de vibración y euforia. Debido a esta atmósfera de placer en la que se desarrolla, el juego tiene un interés intrínseco, canalizando energías hacia un esfuerzo total para lograr su objetivo. Por lo tanto, las actividades recreativas son emocionantes, pero también requieren un esfuerzo voluntario. (...) Las situaciones lúdicas movilizan esquemas mentales. $\mathrm{Al}$ ser una actividad física y mental, el juego desencadena y activas funciones psiconeurológicas y operaciones mentales, estimulando el pensamiento. (...) Las actividades lúdicas integran las diversas dimensiones de la personalidad: afectiva, motora y cognitiva. Por lo tanto, se ve que la actividad lúdica es similar a la actividad artística, como un elemento integrador de los diversos aspectos de la personalidad. El ser que juega y juega es también el ser que actúa, siente, piensa, aprende y se desarrolla (p. 23). 


\section{Las estrategias lúdicas}

Las estrategias lúdicas potencian las actividades de aprendizaje y ayudan a solucionar problemas. Cuando el docente emplea diversas estrategias estas modifican el contenido o estructura de los materiales, con el único fin de facilitar el aprendizaje y comprensión en los niños y niñas (Guerrero, 2014). Por medio de las estrategias lúdicas se promueve a la exploración e indagación en torno a los objetivos, temas, contenidos, además genera un ambiente favorable para que el alumnado sienta interés y motivación por lo que se les está enseñando.

Las estrategias lúdicas contribuyen de manera efectiva el desarrollo cognitivo e integral de los niños y niñas, permitiendo abordar los siete saberes que el ilustre pensador Edgar Morín (1999) considera necesarios para este siglo, pertinentes no como un conocimiento único, sino como una red de conocimiento que lleve a un desarrollo sostenible (Posada, 2014). Los saberes que menciona Morín (1999) son:

a. Las cegueras del conocimiento: el error y la ilusión.

b. Los principios de un conocimiento pertinente.

c. Enseñar la condición humana.

d. Enseñar la identidad terrenal.

e. Afrontar las incertidumbres.

f. Enseñar la comprensión.

g. La ética del género humano.

\section{Importancia de la lúdica en la enseñanza de español}

La lúdica implica el reconocimiento de sí mismo y la relación con el entorno a partir de experiencias placenteras. La relevancia de esta actividad radica en que permite potenciar aspectos relacionados con el pensamiento abstracto, innovador y creativo, de igual forma desarrolla habilidades comunicativas y cooperativas, así como la capacidad de entender problemáticas y buscar posibles soluciones frente a ellas (Castellar, González y Santana, 2015).

La lúdica no solo es un juego como muchos investigadores solían interpretar, también está compuesta por sueños, cuentos, relatos, poesías, imágenes y el uso de símbolos, lo que potencia estructuras mentales, desarrollo de habilidades y destrezas de un individuo, fortaleciendo las relaciones sociales, ya que, desarrolla cualidades como nobleza, generosidad y otras cualidades que propician el trabajo en cooperativo (Posada, 2014).

El juego y el aprendizaje tienen aspectos en común: a) afán de superación; b) la práctica y el entrenamiento que conducen al aumento de las habilidades c) capacidades; d) la puesta en práctica de estrategias que conducen al éxito y ayudan a superar dificultades (Sánchez, 2010). Se infiere la importancia de la lúdica para fortalecer procesos 
de convivencia en la escuela. Al respecto Ascorra et al. (2017) destacan la importancia de identificar estrategias para la administración de las conductas disruptivas. Existen gran variedad de juegos universales de mesa y tablero, de patio y recreo, juegos para reuniones y fiestas o juegos de ingenio y habilidad, que demuestran la importancia y trascendencia que estos han tenido por su aportación al disfrute de la vida, como por el enriquecimiento en las relaciones sociales y en el aprendizaje

A lo largo de la infancia y del primer contacto con la escuela la lúdica se vuelve principal para el desarrollo de distintas habilidades y destrezas que se desarrollan como parte de un proceso dinámico e interactivo. En la etapa inicial la cual se establece entre los 3 y 6 años de edad, las actividades lúdicas se convierten en la cotidianidad de los estudiantes que disfrutan de las mismas.

\section{Estrategias lúdicas en la enseñanza de una lengua extranjera}

El espacio del aula se presenta como un ambiente para distracción, la rigidez y la disciplina; los entornos escolares aburridos promueven el distanciamiento entre estudiantes y profesores. Ante esta situación, es competencia de la institución educativa concebir actividades lúdicas donde los socios de aprendizaje tengan posibilidades de asimilarse y adaptarse más rápido a los contenidos.

La preocupación frecuente permanece sobre el hecho que las instituciones educativas promuevan el distanciamiento de las actividades lúdicas en la experiencia de vida de los alumnos en el aula en lugar de aplicarlo como herramienta facilitadora en el proceso de enseñanza y aprendizaje. Santos (1997) afirma que: " La lúdica es eminentemente educativa en el sentido como está constituida, convirtiéndose en la fuerza impulsora de curiosidad en contraste con el mundo y la vida, siendo el principio de todo descubrimiento la creación“ (p. 45).

De esta manera, las estrategias lúdicas aplicadas en las clases de una lengua extranjera se convierten en un complejo sistema de esquemas de percepción, decisiones, expectativas por parte del docente que piensa ejecutar las mismas. En este caso, Santos (1997) justifica el uso de la lúdica al proporcionar una omisión de la clase tradicional, despertando la curiosidad en los educandos, y eso es lo que promueve el aprendizaje, esa fuerza impulsora establece el descubrimiento respetando el escenario en que está inserto el socio de aprendizaje.

En caso de una lengua extranjera, la comunicación oral es fundamental en el desarrollo pedagógico, a partir de ella es que los jóvenes se expresan permanentemente; sumado a la presencia lúdica en el proceso como estrategia propiciará el ambiente idóneo de disfrute y aprendizaje. Sin embargo, esto no es suficiente para que los jóvenes desarrollen una segunda lengua, el enfrentarse a su contexto y solucionar problemas sería la ruta adecuada en su proceso de aprendizaje de una segunda legua; por esto, se sugiere asumir el juego y utilizar los materiales educativos desde una postura crítica e innovadora para contribuir con la construcción del conocimiento en los estudiantes de español como una segunda lengua en Brasil. Entre muchos pedagogos ha existido la concepción del juego como mediador de procesos para incentivar saberes, generar 
conocimientos y crear ambientes de aprendizaje, mientras que otros han optado por una oposición entre juego y aprendizaje (Duarte, 2003, p. 4).

Los elementos aportados por la lúdica sumado al conocimiento previo de los estudiantes conforman un insumo favorable para la construcción de soluciones desde una perspectiva crítica y amena. Las condiciones como los contextos son cruciales para el desarrollo de lenguaje. Bruner sugirió que el niño aprende a usar el lenguaje para "comunicarse en el contexto de la solución de problemas", en lugar de aprenderlo per se; se enfatiza en el aspecto comunicativo del desarrollo del lenguaje más que en su naturaleza estructural o gramatical (Díaz, 2010, p. 26).

En la elaboración de las estrategias lúdicas a ser aplicadas en las clases de español como lengua extranjera existe primordialmente la necesidad de que el mediador de conocimientos, entienda el anhelo de los estudiantes para que haya comprensión de la importancia de la lúdica; después de esa etapa continua la tarea más ardua referida a la inclusión de las actividades lúdicas, al contenido a ser socializado, sin la extinción de sus esencias siendo sacrificado lo menos posible. Sin embargo, debe haber un equilibrio adecuado entre las colocaciones, donde se obtienen resultados bastante significativos, principalmente con juegos educativos.

\section{Metodología}

Se trata de una investigación cualitativa que permite describir desde la vivencia cotidiana, la comprensión e interpretación de los estudiantes con relación al aprendizaje del español como lengua extranjera, su función en el desarrollo de la mismas; también se reconocen particularidades de los participantes que guían la trayectoria hacia la construcción de conocimientos significativos.

Según Pérez (1994), la investigación cualitativa se considera como un proceso activo, sistemático y rigurosa de indagación dirigida, en el cual se toman decisiones sobre lo investigable en tanto se está en el campo de estudio de interés para el investigador, indica esto que da libertad para la producción de conocimiento en el campo interdisciplinar y transdisciplinar, que evoca la perspectiva naturalista, humanista y la concepción interpretativa del investigador en su misión de conseguir opciones para lograr comprender e interpretar una realidad que es conocida por toda la sociedad.

El estudio tuvo como objetivo verificar las actividades lúdicas socializadas en las clases de español como lengua extranjera. El escenario de intervención fue la Escuela Municipal Popular Remédiense ubicada en el Municipio Nuestra Señora de los Remedios, Estado de Piauí, Brasil; la sección de primer año de educación media estuvo conformada por 16 jóvenes con edades comprendidas entre 14 y 20 años; para la conformación del grupo de interés se seleccionan 8 estudiantes clave, cuya actuación reflejaba poco interés por aprender español como lengua extranjera.

Como técnicas para la recolección de los datos, se utilizan la entrevista semiestructurada a seis de los 8 estudiantes. En ese sentido, se realizó la observación no participante, pues según Tamayo (1995) "es aquella en la que el investigador hace uso de la observación directa sin ocupar un determinado estatus o función dentro de la 
comunidad en la cual se realizará la investigación" (p. 123). a través de instrumentos como el guion de entrevista y el registro de observación. El análisis de la información desde la inferencia permite la comprensión teórica de los elementos identificados.

\section{RESUltados Y DiscusióN}

Después de haber esbozado los elementos teóricos que guían la confrontación de los datos y el análisis de la información, el siguiente apartado contempla los resultados encontrados. En primer momento, se hizo el análisis y revisión de la literatura científica existente sobre las actividades lúdicas; en un segundo momento se inició con la recolección de datos en donde se hizo la aplicación de la guía de entrevistas a los 6 estudiantes que forman parte de grupo de informantes clave; entre las preguntas se encuentran:

1. ¿Cómo te sientes con el uso de actividades lúdicas en la clase de español?

2. ¿Cuál de las actividades lúdicas utilizadas por el profesor en la clase de español, te gustan?

3. ¿Qué herramientas lúdicas mejoran tu comprensión de español en el aula de clases?

4. ¿Los juegos te ayudar a memorizar los contenidos de español en el aula?

5. ¿De qué forma deberían ser desarrolladas las actividades lúdicas en el aula de español?

6. ¿Con qué frecuencia los juegos pueden permitirte tener un mejor aprendizaje social en las clases de español?

\section{Análisis e Interpretación de las Entrevistas}

Las respuestas aportadas por los seis informantes a las preguntas realizadas por los investigadores en el proceso de entrevista se relacionan a continuación:

\section{1 ¿Cómo te sientes con el uso de actividades lúdicas en la clase de español?}

Informante 1: Siento que estoy entendiendo más.

Informante 2: Al principio no me gustaba porque no me gusta hablar con nadie, pero después me empezó a llamar la atención.

Informante 3: Me siento más motivado, antes la profe solo te daba clases con el libro y yo por lo general faltaba los días de clases.

Informante 4: Estoy adaptándome porque es la primera vez que tengo clases dinámicas, mis otros profesores solo explicaban para la prueba.

Informante 5: Me está gustando aprender un poco español, desde que la profe cambió todo, ahora nos divertimos. 
Informante 6: A mí no me gustan los idiomas, siempre me preocupé por inglés, pero desde que la profe entró con actividades dinámicas, me siento que estoy aprendiendo algo para la vida.

\section{Interpretación}

El objetivo principal de esta investigación es valorar las actividades lúdicas socializadas en las clases de española, a partir de la interpretación de los hallazgos, en los siguientes términos:

En torno a las actividades lúdicas, su naturaleza, los hallazgos permiten interpretar el hecho de que ciertamente los profesores que trabajan con español como lengua extranjera se han limitado solo a trabajar con el libro didáctico, impartiendo sus clases de forma tradicional, lo cual generó en el estudiantado una desmotivación y desinterés por adquirir conocimientos en la disciplina.

\section{2 ¿Cuál de las actividades lúdicas utilizadas por el profesor en la clase de español, te gustan?}

Informante 1: Me gusta mucho cuando la profe lleva música y nosotros debemos imitar lo que dice la canción.

Informante 2: A mí me gusta, una dinámica que creó la profa, donde se hace un círculo e imaginamos que vamos para la playa y cada uno de nosotros mete en su mochila un objeto, es muy divertido porque a la final debemos aprendernos lo que llevó cada uno de mis compañeros, se aprende vocabulario.... nunca antes lo había hecho.

Informante 3: Me fascinó cuando la profe iba a enseñar las frutas y nos vendó los ojos, pidió que mordiéramos cada una de las frutas que llevó para saber si la identificamos.

Informante 4: Definitivamente, la profe ha llevado muchas actividades, pero la que impactó y amé fue donde hacíamos dos filas y nos mostraba una palabra y yo debía susurrarla al oído de mi compañero y él al que estaba delante hasta llegar al primero y escribirla en el pizarrón, fue divertido.

Informante 5: Me gustan todas, me mantienen entretenido aprendiendo cosas nuevas y la clase se hace cortísima.

Informante 6: Todas me gustan, pero en especial la carta, que es un juego donde recibes una carta de un amigo de un país y tienes que adivinar cuál es la capital y como se deletrea, es genial.

\section{Interpretación}

Los hallazgos anteriores coligen la idea de la aplicación de elementos lúdicos al interior del aula de clases, y vinculadas con las temáticas abordadas que enriquecen el proceso formativo de los estudiantes; además de acuerdo con la percepción que cada uno tiene, es un factor que motiva su participación en el desarrollo de la clase. La lúdica no solamente tiene aceptación en los adolescentes, se constituye en un atributo que los acerca a las actividades y los motiva a participar de las mimas. 
Informante 1: Cuando la profa coloca videos, me gusta porque me ayuda a entender lo que se dice.

Informante 2: La profa cuando lleva el data show y proyecta unos juegos que son muy divertidos, se necesita de dos participantes para encontrar la palabra correcta.

Informante 3: El cd player con canciones en español de reguetón, aprendo expresiones buenísimas.

Informante 4: Cuando la profe nos envía una palabra en español por el grupo de WhatsApp y cada uno de nosotros debe seguir la letra por la que comienza la palabra, eso me hace pensar y a veces casi que explota mi cerebro porque no me recuerdo, pero es muy buena la actividad.

Informante 5: El video de sigan el líder que la profa proyectó el primer día de clase, me causó mucha risa porque mis compañeros no sabían seguir las instrucciones, pero igual aprendimos la localización de las cosas en el salón de clase.

Informante 6: Los videos y uso del proyector en el aula, se me hace más fácil la comprensión de la lengua extranjera.

\section{Interpretación}

Los hallazgos anteriores permiten ver claramente que la combinación de la lúdica con aplicación de las herramientas en situaciones reales, en torno a la actividad educativa se constituye en un ejercicio interesante y práctico para potenciar y desarrollar nuevas habilidades, que permitan el dominio de español como lengua extranjera. Se puede inferir que los estudiantes son apáticos al modelo tradicional de enseñanza a la rutina, es decir, que se requiere de elementos variados para incentivar su participación. Se evidencia la necesidad de desarrollar espacios lúdicos donde puedan interactuar a través de herramientas o aplicaciones tecnológicas que resultan atractivas y útiles para desarrollar las habilidades, al tiempo de fortalecer aquellos aspectos o temáticas con alguna dificultad para los estudiantes.

\section{$4 \dot{¿}$ Los juegos te ayudan a memorizar los contenidos de español en el aula?}

Informante 1: $\mathrm{Si}$, mucho porque me recuerdo de contenidos que ya había visto pero en verdad ni los recordaba.

Informante 2: Me ayuda, tanto que hasta los practico con mis amigos en la hora de intervalo.

Informante 3: Para mí, ha sido algo maravilloso porque cada vez que la profe habla en español ya muchas palabras conozco porque la aprendí con los juegos.

Informante 4: Claro que me ayuda y mucho, siento que cada día aprendo palabras diferentes.

Informante 5: Para serte sincero, con los juegos he aprendido palabras nuevas y ya no son tan difíciles los exámenes que hace la profe. 
Informante 6: Siiiiiiiii, hasta podría contar la cantidad de palabras que he aprendido, ahora hasta veo televisión en español y entiendo bastante.

\section{Interpretación}

Los hallazgos anteriores permiten evidenciar lo efectivo que han sido los juegos en las clases de español, se observó en los estudiantes gran acogida por el trabajo aplicado por la profesora, y se notó un esfuerzo importante por aprender en cada actividad recreativa, notándose un aprendizaje significativo que permitió fortalecer en los estudiantes los contenidos temáticos, demostrando lo mucho aprendido al jugar en las clases.

5 ¿De qué forma deberían ser desarrolladas las actividades lúdicas en el aula de español?

Informante 1: Por mí, en forma colectiva porque aprendo de las personas más inteligentes.

Informante 2: A mi gustaría que sea en equipos y en cada aula se mezclen los equipos de manera que podamos compenetrarnos cada día más a través del idioma.

Informante 3: Seria genial como hasta ahora se ha trabajado en pareja, en tríos y hasta en grupos de 5 personas.

Informante 4: Como sea menos que yo tenga que trabajar solito.

Informante 5: En forma colectiva, se aprende y se disfruta compartir con los colegas.

Informante 6: En grupos porque me ayuda cuando no entiendo algunas palabras.

\section{Interpretación}

Los hallazgos anteriores permiten evidenciar que el trabajo cooperativo y colaborativo es la clave para mejorar y aprender la lengua extranjera, se deja entrever que cuando se aplican actividades con la finalidad de cooperar, el estudiante con mayor conocimiento puede nutrir al otro que no entiendo o desconoce el significado de la palabra en el momento.

6 ¿Con qué frecuencia los juegos pueden permitirte tener un mejor aprendizaje social en las clases de español?

Informante 1: Siempre

Informante 2: Siempre

Informante 3: Siempre

Informante 4: Siempre

Informante 5: Siempre

Informante 6: Siempre

\section{Interpretación}

Estos resultados permiten evidenciar como los juegos favorecen el desarrollo de habilidades relacionadas con los comportamientos en sociedad, estimulando a menudo 
la sociabilidad y reacciones afectivas, cognitivas, morales, culturales y lingüísticas. El uso de juegos según los estudiantes siempre ayuda en los factores de integración social permitiéndoles tener un mejor aprendizaje en la clase de español.

\section{Conclusiones}

El uso de actividades lúdicas estimula la creatividad; se deja de lado la clase habitualmente tradicional; la lúdica como recurso didáctico genera mayor interés y despierta en el estudiante el deseo de participar al involucrarse en las diferentes tareas buscando incrementar su conocimiento.

Por tanto, se cree que las actividades lúdicas utilizadas en la escuela municipal Popular Remédiense pudieron cooperar para que el aula de primer año de educación media se transformará en un recinto recreativo de conocimientos o en un lugar apacible y motivador, ya que, por medio de las actividades lúdicas el estudiante tuvo la posibilidad de explorar su creatividad de manera más amplia, mejorando su seguridad, alcanzando un máximo aprovechamiento de las clases.

La lúdica representa una estrategia para estimular la creatividad, la confianza y la interacción social entre los estudiantes; se incrementa la participación de los estudiantes en las actividades propuestas por docentes. El adolescente crea una resistencia en la institución educativa y en su enseñanza escolar, puesto que la mediación de los aprendizajes es muy monótona y está muy lejos de ser, lo que es una enseñanza lúdica.

También se logró observar que, durante los intercambios de saberes, hubo una participación masiva por parte de los estudiantes en las diferentes actividades que les presentaba el profesor. por eso es de suma importancia el uso de las herramientas lúdicas de forma planificada.

Se puede concluir que las actividades lúdicas aportan beneficios en la apropiación de conocimientos, ya que, los estudiantes se involucran, participando y motivándose en el aprendizaje de español como lengua extranjera. Pero además de los elementos lúdicos también se relacionaron las actividades con temáticas académicas que contribuyen al mejoramiento del desempeño académico estudiantil.

Finalmente, con relación a las actividades lúdicas se logró vincular a los estudiantes no solo con el aprendizaje de vocabulario enfocado a las diferentes temáticas que presenta el libro didáctico, sino que tomaron sentido de pertenencia por aquellas palabras que forman parte de su día a día.

\section{REFERENCIAS}

Ascorra, P., Vásquez, P., Passalacqua, N., Carrasco, C., López, V., Núñez, C. y Álvarez, M. (2017). Discursos en torno a la administración del conflicto y su relación con la convivencia escolar. Cultura Educación y Sociedad, 8(2), 21-42. https://doi. org/10.17981/cultedusoc.8.2.2017.02

Calderón, L., Marín, S. y Vargas, N. (2015). La lúdica como estrategia para favorecer el proceso de aprendizaje en niños de edad preescolar de la institución educativa 
Nusefa de Ibagué. [Trabajo grado]. Universidad del Tolima, Colombia. Disponible en http://repository.ut.edu.co/handle/001/1409

Castellar, G., Gonzalez, S. y Santana, Y. (2015). Las actividades lúdicas en el proceso de enseñanza aprendizaje de los niños de preescolar del instituto madre teresa de Calcuta. [Trabajo grado]. Universidad del Tolima, Colombia. Disponible en https:// repositorio.unicartagena.edu.co/handle/11227/2106

Díaz, L. (2010). La lúdica como estrategia de aprendizaje en el desarrollo de habilidades del inglés en niños y niñas de grado PBP. [Tesis magistral]. UNAM, Colombia. Recuperado de http://www.bdigital.unal.edu.co/41019/1/04868267.2014.pdf

Duarte, D. (2003). Ambientes de aprendizaje: una aproximación conceptual. Estudios pedagógicos (Valdivia), (29), 97-113. https://dx.doi.org/10.4067/S071807052003000100007.

Guerrero, R. (2014). Estrategias lúdicas: herramienta de innovación en el desarrollo de las habilidades numéricas. Revista Electrónica de Humanidades, Educación y Comunicación Social, 18(9), 30-43. Disponible en http://ojs.urbe.edu/index.php/ redhecs/article/view/2427

Morín, E. (1999). Los siete saberes para la educación del futuro. Francia: Unesco.

Pérez, G. (1994). Investigación cualitativa. Retos e interrogantes. Madrid: La Muralla.

Posada, R. (2014). La lúdica como estrategia didáctica. [Tesis magistral]. UNAM, Bogotá, D.C. Recuperado de http://www.bdigital.unal.edu.co/41019/1/04868267.2014. pdf

Sánchez, G. (2010). Las estrategias de aprendizaje a través del componente lúdico. Marco ELE: Revista de Didáctica Español Lengua Extranjera, (11), 1-68. Disponible en https://marcoele.com/suplementos/estrategias-y-componente-ludico/

Santos, S. (2006). Educação, arte e jogo. Petrópolis: Vozes.

Santos. S. (1997). Lúdico na Formação do Educador. Petrópolis-RJ: Vozes.

Silveira, R. \& Barone, D. (1998). Jogos Educativos computadorizados utilizando a abordagem de algoritmos genéticos. Sao Paulo: Universidade Federal do Rio Grande do Sul.

Tamayo, M. (1995). El proceso de la investigación científica. (3 Ed.). México, D.F.: Limusa.

Teixeira, C. (1995). Ludicidade na Escola. São Paulo: Loyola.

Zúñiga, G. (noviembre, 1998). La pedagogía lúdica: una opción para comprender. En, FUNLIBRE, V Congreso Nacional de Recreación. Coldeportes Caldas, Universidad de Caldas, FUNLIBRE, Manizales, Caldas, Colombia.

Ramón Antonio Hernández Chirinos de Jesus obtuvo su licenciatura en la Universidad del Zulia (Venezuela), especialización en la Universidad Católica Cécilio Acosta (Venezuela), Doctorado en la Universidad Experimental de las Fuerzas Armas, las especialidades en Brasil en la Facultad evangélica de Piauí (Brasil). Es profesor invitado por la Universidad Autónoma de Juarez. Tabasco (México), profesor jubilado del Ministerio de Educación de Venezuela. Profesor contratado por la Universidad del Zulia (Venezuela). Profesor invitado por la Universidad Estadual de Piauí desde 2016 
hasta el 2019, Profesor Coordinador del área de inglés en el Municipio de Porto (Piauí, Brasil). https://orcid.org/0000-0002-0663-8983

Francisco das C Silva de Jesus Hernández obtuvo su licenciatura en la Universidad del Estadual de Piauí (Brasil), especialización en la Universidad Estadual de Piauí (Brasil). Profesor de Lared estadual de Piauí, profesor de la red municipal de Porto (Piauí, Brasil), Secretario de Asistencia social en Porto (Piauí, Brasil). https:// orcid.org/0000-0002-5420-0679 